Original Research Paper

\title{
Mental Health, Quality of Life and Medication Use Among Care Home Residents and Community Dwelling Older People
}

\author{
${ }^{1}$ Marie O'Neill, ${ }^{1}$ Assumpta Ryan, ${ }^{2}$ Paul Slater, ${ }^{3}$ Finola Ferry and ${ }^{3}$ Brendan Bunting \\ ${ }^{1}$ School of Nursing and Institute of Nursing and Health Research, \\ Ulster University, Northland Road, Derry, Co Londonderry BT48 7JL, Northern Ireland, UK \\ ${ }^{2}$ School of Nursing and Institute of Nursing and Health Research, \\ Ulster University, Shore Road, Newtownabbey, Co. Antrim, BT37 OQB, Northern Ireland, UK \\ ${ }^{3}$ School of Psychology and Psychology Research Institute, \\ Ulster University, Cromore Road, Coleraine BT52 1SA, UK
}

\author{
Article history \\ Received: 27-09-2019 \\ Revised: 11-11-2019 \\ Accepted: 02-01-2020 \\ Corresponding Author: \\ Marie O'Neill \\ School of Nursing and Institute \\ of Nursing and Health \\ Research, Ulster University, \\ Northland Road, Derry, Co \\ Londonderry BT48 7JL, \\ Northern Ireland, UK \\ Email: m.oneill@ulster.ac.uk
}

\begin{abstract}
In most countries, the prevalence of depression in care homes is substantially higher than among community-dwelling older people. Negative media reports and the stigma associated with long-term care perpetuates the belief that community care is associated with a better quality of life than nursing or residential home care. The aim of this paper is to investigate mental health, quality of life and medication use among a sample of care home residents and community dwelling older people. This cross-sectional study comprised of structured interviews with 75 care home residents and 852 community dwelling older adults in a large health and social care trust within the U.K. Data were collected using an abridged version of the World Mental Health-Composite International Diagnostic Interview (WMH-CIDI). Health-related quality of life was assessed using the SF-6D, derived from responses to the SF-12, which was embedded within the screening section of the WMH-CIDI. Results showed a significantly lower level of mental disorders among older people in the care home sample. Health-related quality of life results indicated that although care home participants were significantly more limited in their physical functioning, they reported better mental health and social functioning than older people living in the community. These results were statistically significant. Prescription rates for mental health medication were higher in the care home sample. This study provides tentative findings about mental health and quality of life in care homes, which should be further explored in future research. The study addresses the negative public perception of quality of life in care homes while contributing to the debate around the health care implications of 'ageing in place' for older people. There is a need to address the relationship between mental health, quality of life and medication use among older people across a range of care settings.
\end{abstract}

Keywords: Older People, Mental Health, Care Home, Quality of Life, Medication

\section{Introduction}

The ageing population of the world has given rise to a changing philosophy surrounding the provision of health and social care from institutional to community-based care. This shift is aimed principally at helping people to live in their own homes for as long as possible. However, the need for residential care is expected to increase due an ageing population, changes in family structure and the impact of dementia and other age-related conditions. While research has focused on the physical well-being of older people, the mental health of older people remains a relatively under-researched topic. Moreover, little is known about the mental health and quality of life of older people living in long-term care settings in comparison to their community dwelling peers. 


\section{Ageing and Mental Health}

The relationship between age and mental health has been widely researched in the literature. Some studies have reported decreased prevalence rates for mental health disorders in older age (Scott et al., 2008; Bunting et al., 2012; Karel et al., 2012; Reynolds et al., 2015). Others have reported lifetime and current prevalence rates of mental disorders in older people ranging from 1 to $18 \%$ (Volkert et al., 2013; Reynolds et al., 2015). The World Health Organization (WHO, 2015) estimated that over $20 \%$ of adults aged 60 years and over, experience a mental or neurological disorder. The most common neuropsychiatric disorders in this age group are depression (7\%); dementia (5\%) anxiety disorders which affect $3.8 \%$ and substance use problems which affect almost $1 \%$ of the older population (WHO, 2015). A meta-analysis of mental disorders in older adults in Western Countries undertaken by Volkert et al. (2013) found the lifetime prevalence of affective disorders was $16.52 \%$, anxiety disorders $2.63 \%$, substance abuse disorders $11.71 \%$ and psychosis $4.7 \%$

Depression is a key mental health issue in later life and is associated with physical co-morbidities, lower quality of life and disability (Covinsky et al., 2010; Naylor et al., 2012). A systematic review and metaanalysis of 24 studies on the prevalence of depression in later life (75 years and older) found that the prevalence of major depression ranged from $4.6 \%$ to $9.3 \%$ and that of depressive disorders from $4.5 \%$ to $37.4 \%$. (Luppa et al., 2012). According to the Royal College of Psychiatrists (RCPsych), depression may affect one in five older people living in the community and two in five older people living in care homes (RCP, 2014). A crosssectional study of community dwelling people aged 60 years or older in Brazil $(\mathrm{n}=1563)$ found that nearly $3.8 \%$ to $15 \%$ of participants presented with depressive symptoms (Barcelos-Ferreira et al., 2013). A systematic review by Seitz et al. (2010) on the prevalence of psychiatric disorders in nursing homes reported prevalence rates for depression ranging from $4 \%$ to $25 \%$ for major depressive disorder and $29 \%$ to $82 \%$ for minor depression or the presence of depressive symptoms. A Dutch study exploring the incidence of depression in a sample of nursing $(\mathrm{n}=1324)$ and residential $(n=1,723)$ home residents reported that the incident rate was 13.6 per100-person years in the nursing home and 10.2 per 100-person years in the residential home (Boorsma et al., 2012).

\section{Psychotropic Medication Use}

The use of psychotropic drugs in European care homes is reported to be common, with prescription rates of psychotropic drugs higher in institutionalized older population than that of community dwelling older people
(Azermai et al., 2011; Haasum et al., 2012). Moreover, it has been determined that the prescription rate of psychotropic medication is on the increase in care homes (Ruths et al., 2013). Psychotropic agents including antipsychotics, antidepressants and/or anxiolytics are often prescribed as the first-choice treatment option for mental health conditions including sleep disturbance, anxiety, depression even though professional guidelines advocate non-pharmacological management of symptoms (Pitkala et al., 2015; Zuidema et al., 2015). Underuse as well as overuse of psychotropic medication have also been reported in this population (Hanlon et al., 2011). Moreover, a recent study on the use of psychotropic medications in care homes identified potential benefits of antipsychotic medication reduction (Simmons, 2017).

An evaluation of the quality of antidepressant management and prescribing patterns in nursing homes in the USA showed a discrepancy between prescriptions of antidepressants and the presence or absence of depression diagnosis. A review of 205 medical charts indicated that for those with documented depression diagnoses $(n=126)$, nearly one quarter were not prescribed antidepressants. Moreover, out of 79 medical chart reviews indicating no depression diagnosis, nearly a third were receiving an antidepressant (Shah et al., 2011). Another American study of nursing home residents identified that second-generation antipsychotics were commonly used for off-label indications, including treatment of bipolar disorder, depression and psychotic symptoms; and these indications were not always evidence-based (Kamble et al., 2010). In the United Kingdom, it is estimated that approximately $21 \%$ of care home residents are receiving antipsychotic medications and that more than $80 \%$ of this prescribing is for residents without a diagnosis of severe mental illness (Shah et al., 2011). Significantly, Harrison et al. (2018) found that an increased number of psychotropic medications were associated with lower quality of life scores. Furthermore, due to ageing co-morbidities and the need for the prescription of multiple medications, older people may experience adverse drug effects, drug interactions, forgetfulness, lack of familial and social support and care and may not be fully concordant with taking long term medications. Tsai et al. (2012) stated that in general only $50 \%$ of the general population has been estimated to adhere to their medications and this may range from 47 to $100 \%$ in older people. In addition, medication concordance has been positively correlated with educational status and individual's awareness of diseases and prescribed medications (Shruthi et al., 2016).

\section{Quality of Life (QoL)}

There is no standard definition of QoL and conceptualizations of QoL vary considerably (Dichter et al., 
2013). The WHO defines QoL as “individual's perception of their position in life in the context of the culture and value systems in which they live and in relation to their goals, expectations, standards and concerns" (WHO, 1997, p. 1). Several studies have investigated which QoL domains older people deem as having high importance. Results have identified a broad range of QoL priorities including health, activities of daily living, sensory abilities and mobility (Kalfos and Halvorsrud, 2009; Molzahn et al., 2011); the home environment and family (Beaumont and Kenealy, 2004; Bowers et al., 2009; James et al., 2014) and finances and social life (Evans et al., 2005). Furthermore, Bradshaw et al. (2012) identified four key factors influencing QoL in care homes; the person's acceptance and adaptation to their living situation, their 'connectedness' with others, living in 'a homelike environment' and carers displaying 'caring practices' Similar themes emerged from the study by Bowling et al. (2013) who identified psychological well-being and a positive outlook, having health and functioning, social relationships, leisure activities, neighbourhood resources, adequate financial circumstances and independence as key indicators of QoL in old age.

The Northern Ireland Study of Health and Stress (NISHS) (Bunting et al., 2012) was part of a series of epidemiological studies of mental health, conducted in over 30 countries throughout the world under the auspices of the World Health Organisation (WHO). Results indicated that lifetime prevalence rates for mood and anxiety disorders increased with age, dropping substantially for the 65+ group. Epidemiological studies of mental health have largely ignored the growing population of older people in residential and nursing homes, so it is difficult to determine the extent to which their mental health needs are met. Despite current demographic trends and the dearth of reliable information about the extent and impact of mental health problems among care home residents, this group was excluded from the NISHS and from all studies involved in the WHO initiative. From the authors' perspectives, this constituted a 'missed opportunity' and provided the impetus for the present study which aimed to examine mental health, quality of life and medication use among a sample of care home residents and community dwelling older people.

\section{Methods}

\section{Participants and Selection Procedure}

This paper reports on the comparison of two samples of older people, who were administered the same structured survey instrument, the World Mental HealthComposite International Diagnostic Interview (WMHCIDI) (Kessler and Üstun, 2008).

\section{Community Sample}

The sample of 852 individuals aged 65 and over living in the community was drawn from the Northern Ireland Study of Health and Stress (NISHS). The NISHS (Mc Lafferty et al., 2018) was part of a series of World Mental Health Survey Initiative epidemiological studies of mental health, conducted in over 30 countries throughout the world under the auspices of the World Health Organisation (WHO). The study was a representative household survey of English speakers, aged 18 years and older. Participants were selected from a multistage, clustered, area probability sample of households. To achieve an equal probability sample, a three-stage area sample design was used. First, electoral wards were selected from each local government district, based on probability proportionate to size. Two-census output areas (COAs) were then selected per ward and finally a sample of 10 dwellings were selected from each COA. The total NISHS sample comprised data from 4,340 adults, including 852 people aged 65 years and over that were included in the current study.

\section{Care Home Sample}

The sample of 75 care home residents was drawn from nursing and residential homes in a Health and Social Care Trust in a region of the United Kingdom (UK). In the initial stages of data collection, 20 care homes were randomly selected from a list provided by the statutory body responsible for the regulation of care homes. To obtain the necessary sampling frame, the manager of each selected care home was asked to list all residents who met the following selection criteria: aged 65 years of age; minimal cognitive impairment as defined by the Mini Mental State Examination (Folstein et al., 1975) and physically able and willing to consent and participate in the study. From this population, a number of individuals from each care home were selected using proportional allocation based on the number of residents who met the selection criteria within each home. Sample selection was undertaken by two members of the research team using Kish tables on care homes listings (Kish, 1965). The researcher then met with potential participants to provide verbal and written information about the study and to answer any questions about the study. Residents who wished to take part were then asked to sign a consent form for the interview, which was arranged at a time and place to suit the resident. The initial interviews were recorded and checked to ensure the rigor of the data collection procedures. This process also determined the acceptability of questions and interview duration for care home residents. The selected respondents were interviewed by the first author who is 
an experienced qualitative researcher and mental health nurse. The interview responses were recorded on a laptop. Interview questions were also presented in a respondent booklet $(\mathrm{RB})$ that provided a visual aid as the questions were being read by the interviewer. Misunderstandings based on vagueness of terms were addressed by introducing clarifications and examples. The use of such instruction aimed to improve the quality of data obtained.

\section{Instruments}

The World Mental Health-Composite International Diagnostic Interview (WMH-CIDI) (Kessler and Üstun, 2004) was used to collect data. WMH-CIDI is a widely used and extensively tested interview schedule used for the assessment of mental health disorders. Lay interviewees underwent the standard WHO WMH-CIDI training program which included 30 hours of at-home self-study with a series of manuals and CD-ROMs followed by 3-5 days of in-person training by skilled CIDI Trainers. A compulsory assessment was undertaken at the end of training to award certification which was necessary to undertake interviews. CIDI training included data collection, editing, coding and clearing in addition to study specific interviewing techniques and field quality control issues. The CIDI interview contains questions on the factors influencing mental health including depression, anxiety disorders, chronic illness and social networks. The interview generates psychiatric diagnoses according to the definitions and criteria of both the International Statistical Classification of Diseases tenth edition (ICD10) (WHO, 1992) and the Diagnostic and Statistical Manual of Mental Disorders Fourth Edition (DSM-IV) (American Psychiatric Association, 1994). The CIDI allows the investigator to measure the prevalence of mental disorders; the severity of these disorders in addition to assessing medication use and treatment interventions. The WMH-CIDI also establishes symptoms and age of onset, data which will enable the research team to determine whether reported symptoms commenced prior to, simultaneous with, or following, admission to a care home. Embedded within the WMHCIDI are instruments assessing health related quality of life and medication use, as well as a range of questions relating to socio-demographic characteristics. The WMH-CIDI in its entirety takes an average of approximately 1.5 to 2 hours to administer. However, the interview time varies widely depending on the number of diagnostic sections for which the respondent screens positive. For this study, face- to face structured interviews were undertaken by the principal investigator using an abridged electronic CIDI instrument and took approximately 1.5 hours to complete.

\section{Reliability and Validity}

A considerable amount of research has been undertaken by researchers to refine the instrument prior to the WMH Survey Initiative (Brugha et al., 2001; Kessler and Ustun, 2004). The validity of the WMHCIDI has been tested to determine whether the diagnoses generated by the WMH-CIDI are consistent with those obtained independently by trained clinical interviewers such as psychiatrists. Results have shown that CIDI diagnoses are significantly related to independent clinical diagnoses (Kessler and Ustun, 2004).

\section{$S F-12 v 2$}

The Short Form 12 (SF-12) is a shortened form (12 items) of the SF-36v2 Health Survey. It is a generic assessment of health-related quality of life (HR QOL) from the client/patient's perspective. The instrument contains 12 questions, addressing eight key domains of health related QoL: physical functioning, limitation of role due to physical health problems, bodily pain, general health vitality, social functioning, role limitations due to emotional problems and finally general mental health. These eight domains can be summarised into a physical component summary (PCS) and a mental component summary (MCS. The SF-12 was also used to derive a preference based index of health related QoL for each individual (a quality of life weighting ranging from 0 to 1 ). A scoring algorithm developed by Brazier and Roberts (2004) was used to transform SF-12 response levels into SF-6D response levels. The SF-6D is also a generic health related quality of life (QoL) survey which assesses six dimensions of health: physical functioning, role limitations, social functioning, pain, mental health and vitality.

\section{Outcome Measures}

The current paper considered four broad outcome measures: (1) Lifetime mood and anxiety disorders; (2) 12-month mental health medication use; (3) overall health related quality of life and (4) individual health related quality of life domains. The prevalence of any lifetime mood or anxiety disorder was determined by the percentage of the study sample that met the DSMIV criteria for a mood or anxiety disorder at some point their lifetime, as assessed by the WMH-CIDI. This summary variable was based on the assessment of 13 mood and anxiety disorders: major depressive episode, major depressive disorder, dysthymia, specific phobia, social phobia, panic disorder, obsessive-compulsive disorder, generalised anxiety disorder, separation anxiety disorder, adult separation anxiety disorder, agoraphobia with and without panic and post-traumatic stress disorder. A dichotomous variable was created to 
indicate whether an individual met the criteria for any of these disorders during their lifetime. All participants were asked about medication they had taken in the previous 12-months for problems with 'emotions, nerves or mental health' within the following broad medication categories: sleeping pills or other sedatives, anti-depressants, hypnotics or anxiolytics and anti-psychotics. In addition, the care home participants consented to provide details of medication records since their admission to the care home. A dichotomous variable was created to indicate if an individual had taken any of these medication types in the previous 12 months.

Health-related quality of life was assessed using the SF-6D, derived from responses to the SF-12, which was embedded within the screening section of the WMHCIDI. A scoring algorithm developed by Brazier and Roberts (2004) was used to transform SF-12 response levels into SF-6D response levels. The SF-6D is a classification system for describing health related quality of life comprising six multi-level dimensions: physical functioning, role limitations, social functioning, pain, mental health and vitality. Response levels for each of the dimensions indicate the level of difficulty experienced by participants. There are three response levels for physical function, four for role limitations and five response levels for each of the other four dimensions. Higher response levels indicate poorer outcomes. An overall health-related quality of life index was also derived from SF-6D responses. Population based weights, also derived by Brazier and Roberts (2004), were applied to responses to determine each individual's health-related quality of life weighting (from 0-1). The resulting index describes a potential 18,000 health states in all, with higher scores indicating better overall health-related quality of life.

\section{Data Analysis}

Statistical algorithms, developed in Harvard for use in all WMH surveys, were applied to the final dataset to generate a series of DSM-IV (APA, 1994) dichotomous diagnostic variables for mental health disorders. Lifetime prevalence of any mood or anxiety disorder was determined by calculating the percentage of participants who met the criteria for each any of the 13 mood or anxiety disorders, detailed previously at any point in their lifetime. Descriptive statistics were used to estimate lifetime mood or anxiety disorders, medication use, average overall health-related quality of life scores and dimension scores. Independent samples t-tests and chisquared tests were used to compare outcomes between community dwelling and care home residents. Linear regression analyses (Hosmer and Lemeshow, 1989) were used to examine the association of sex, setting (community versus care home) and age with any lifetime mood or anxiety disorder. Logistic regression was similarly used to examine the association of these variables with any 12-month medication use for problems with 'emotions, nerves or mental health'. Odds ratios indicated the strength of these associations and statistical significance was evaluated using 2-sided, design-based P.05-level tests. The study examined the association of sex, setting and age with overall healthrelated quality of life (SF-6D score) using linear regression. Where assumptions of equal variance are violated, tests that deal with unequal variances (e.g. Welch's test for unequal variance) were used.

\section{Results}

\section{Profile of Participants}

Of the 927 participants aged 65 years and over, 411 $(44.3 \%)$ were males and 516 females $(55.7 \%)$. There were $852(91.9 \%)$ living in the community and $75(8.1 \%)$ living in care homes. The male/female breakdown varied across the two settings, with a $46.2 / 53.8 \%$ male/female breakdown in the community sample and $22.7 / 77.3 \%$ breakdown in the care home sample.

\section{Prevalence of Any Lifetime Mental Health Disorder and Use of Mental Health Medication}

DSM-IV disorders were lowest amongst the 65+ year age group, (22.2\%) community care sample in comparison to (12\%) in the care home sample. Results show a significant difference in the prevalence of any lifetime mental health disorder between the two samples, with a much higher level among the community sample (X2 $=9.93,1 \mathrm{df}, \mathrm{p}=0.002)$. While $18.0 \%$ of individuals living in the community met the criteria for any lifetime mood or anxiety disorder, just $3.0 \%$ of those in care homes had a lifetime disorder. panic disorder and panic attacks compared to $2.8 \%$ reporting lifetime panic disorder in the NISHS. The lifetime prevalence of Posttraumatic Stress Disorder (PTSD), (6.0\%) and specific phobia, (SP) (5.6\%) were higher among the NISHS sample compared to the care home sample (PTSD 1.3\% $(\mathrm{n}=1)$ and Specific Phobia $2.6 \% \mathrm{n}=2)$. A similar picture emerged for lifetime major depressive disorder which was prevalent among $2.6 \%(\mathrm{n}=2)$ of the care home sample compared to $8.1 \%$ of the NISHS sample. Results indicate that overall there was a very low prevalence of DSM disorders among the care home sample. CIDI data to show participants who had met the DSM diagnostic criteria for mental health disorders are outlined in Table 1. 
Table 1: Lifetime prevalence of DSM-IV disorders

\begin{tabular}{|c|c|c|}
\hline Disorder & Community \% & Care Home \% \\
\hline \multicolumn{3}{|l|}{ Anxiety disorders } \\
\hline Panic disorder & 1.8 & 2.6 \\
\hline Panic attacks & Not measured & 2.6 \\
\hline GAD without hierarchy & 2.8 & 0.0 \\
\hline Social phobia & 2.1 & 0.0 \\
\hline Specific phobia & 5.6 & 2.6 \\
\hline Agoraphobia without panic & 0.9 & 0.0 \\
\hline Post-traumatic stress disorder & 6.0 & 1.3 \\
\hline Obsessive compulsive disorder & 0.0 & 0.0 \\
\hline $\mathrm{SAD} / \mathrm{ASA}$ & 2.0 & 0.0 \\
\hline Any anxiety & 16.1 & 0.0 \\
\hline Mood disorders MDD with hierarchy & 8.1 & 2.6 \\
\hline Dysthymia with hierarchy & 0.9 & 0.0 \\
\hline Bipolar-broad & 0.4 & 0.0 \\
\hline Any mood & 9.0 & 0.0 \\
\hline \multicolumn{3}{|l|}{ Impulse-control disorders } \\
\hline ODD with hierarchy & 0.5 & 0.0 \\
\hline Conduct disorder & 0.2 & 0.0 \\
\hline ADHD & 0.4 & 0.0 \\
\hline IED with hierarchy & 1.2 & 0.0 \\
\hline Any impulse & 1.9 & 0.0 \\
\hline \multicolumn{3}{|l|}{ Substance disorders } \\
\hline Alcohol abuse w/without dependence & 3.6 & 0.0 \\
\hline Alcohol dependence with abuse & 1.2 & 0.0 \\
\hline Drug abuse w/without dependence/Abuse & 0.0 & 0.0 \\
\hline Any substance & 3.6 & 0.0 \\
\hline Any disorder & 22.2 & 12.0 \\
\hline
\end{tabular}

GAD, Generalized anxiety disorder; SAD/ASA, separation anxiety disorder/adult separation anxiety; MDD, major depressive disorder; ODD, oppositional-defiant disorder; ADHD, attention-deficit hyperactivity disorder; IED, intermittent explosive disorder.

Table 2: Comparison of 12-month mental health medication use among community and care home dwellers

\begin{tabular}{lll}
\hline & Community sample \% (n) & Care home sample \% (n) \\
\hline Sleeping pills or other sedatives $\dagger$ & $9.0(77)$ & $41.8(28)$ \\
Antidepressants $\dagger$ & $5.4(46)$ & $28.4(19)$ \\
Hypnotics/Anxiolytics $\dagger$ & $4.0(34)$ & $20.9(14)$ \\
Anti-psychotics $\dagger$ & $0.1(1)$ & $6.0(4)$ \\
Any Mental Health Medication $\dagger$ & $14.7(125)$ & $64.2(43)$ \\
\hline
\end{tabular}

$\dagger$ Indicates significant differences between community and care home dwellers at the 5\% level

Table 2 summarises the percentage of individuals who were taking specific medication types and shows that, despite low levels of mental disorders among the care home sample, medication prescription levels were considerably higher than those recorded for community dwelling older adults. Table 2 shows that $41.8 \%$ of the care home sample had taken sleeping pills or other sedatives in the previous 12 months compared to $9.0 \%$ of those over 65 years old living in the community. Displaying a similar trend, $28.4 \%$ of the care home sample were on anti-depressant medication in the previous 12-months compared to $5.4 \%$ of the community sample. Almost $21 \%$ of those living in care homes had taken hypnotics or anxiolytics in the previous 12 months compared to $4.0 \%$ of older adults in the community. Finally, a higher percentage of care home residents had taken anti-psychotic medication compared to those in the community (6.0\% compared to $0.1 \%)$. These findings are noteworthy insofar as it appears to run contrary to the results presented earlier in the paper, which showed lower levels of mental disorders among care home residents compared to older adults within the general population.

Considering all four mental health medication types, a comparison of the percentage of individuals that took any mental health medication in the previous 12 months for mental health problems again shows significant differences between the care home and community sample. Chi-square tests show that those living in care homes were significantly more likely to have taken medication for mental health problems in the previous 12 months $(\mathrm{X} 2=101.912,1 \mathrm{df}, \mathrm{p}=0.000)$. Within the community $14.7 \%$ of individuals reported that they had taken a mental health medication in the previous 12 months compared to $64.2 \%$ in the care home sample. 


\section{Association of Setting, Gender and Age with Mental Health and Medication}

Logistic regression analyses were used to examine the association of setting, sex and age with any DSMIV lifetime disorder and the use of any mental health medication in the previous 12 months (Table 3). While individuals living in care homes were significantly less likely to meet the criteria for a lifetime mental health disorder, they were significantly more likely to have taken mental health medication in the previous 12 months. Females were more likely than males to have a lifetime mental health disorder and more likely to have taken a mental health medication. Older individuals were less likely to have a lifetime mental disorder, while there was no association between age and mental health medication.

\section{Comparison of SF12 Scores}

The SF-12 was scored so that a high score indicates better functioning. The Physical Component Summary (PCS) and the Mental Component Summary (MCS) scores have a range of 0 to 100 and are designed to have a mean score of 50 and a standard deviation of 10 . Thus, scores greater than 50 represent above average health status. Response levels for each of the six dimensions are described in the methods section, but to summarise, higher response levels indicate poorer outcomes for each of the dimensions.

Table 4 presents the results from an independent samples $\mathrm{t}$-test, assuming unequal variances, found a significant difference in of health-related quality of life, with higher scores indicating worse outcomes, individuals living in care homes had significantly poorer physical functioning $(\mathrm{p}=0.000)$ and were more limited in their daily roles $(p=0.044)$ compared to those living in the community. Conversely, those living in care homes had better outcomes relating to social functioning $(\mathrm{p}=0.000)$, pain $(\mathrm{p}=0.001)$ and mental health $(\mathrm{p}=$ 0.001 ). There were no significant differences in relation to vitality between the two sub-samples overall health related quality of life $(p=0.028)$ and in all dimensions of health-related quality of life, except for 'vitality'.
Overall, health-related quality of life was poorer among the sample of individuals living in care homes. Considering each of the 6 dimensions.

Overall the total SF12 scores were statistically different across the settings with NISHS (65+) having lower scores overall. Table 3 shows that NISHS sample had an MCS mean score of 54, compared to a mean score of 61.6 for the care homes sample. The NISHS had a mean PCS score of 43.8, while the mean PCS score for the care homes sample was 35 . The mean scores for the US general population for this age group were 43.5 for the PCS and 51.5 for the MCS.

Multiple regression was conducted to examination of the impact of independent variables (gender, age, setting and physical health scores) show that the model explains $7.9 \%$ of the variance of mental health scores. Analysis of variance scores show the significance of the model in predicting mental health $\mathrm{F}(4,992)=20.753, \mathrm{p}=0.001)$. Examination of the impact of the four independent variables show that as age increased by a year, mental health scores increased by 0.12 , gender (male to female) 0.063; setting (at NISHS Vs Care Home) 0.229, mental health increased by 0.112 with each one-unit in physical health and all at a statistically significant level $(\mathrm{p}=0.001)$.

Similar analysis was run with physical health show that the model explains $7.5 \%$ of the variance. Analysis of variance scores show the significance of the model in predicting physical health $\mathrm{F}(4,922)=19.8, \mathrm{p}=0.001)$. Examination of the impact of the four independent variables show that as age increased by a year, physical health scores decreased by -0.146 , gender (male Vs female) -0.107 , setting (at NISHS Vs Care Home) 0.159 , physical health component increased by 0.112 with each one-unit in mental health and all relationships at a statistically significant level $(\mathrm{p}=0.001)$.

Further examination of physical health component shows that, after controlling for age, only NISHS (65+) and the Care Homes had a statistically significant difference in mean scores (Table 4). Likewise, with mental health component, mean scores were different at a statistically significant level however the means scores were significantly higher in the NISHS (65+) group.

Table 3: Logistic regression analyses examining association of setting, gender and age with mental health and medication

\begin{tabular}{llcc}
\hline \multicolumn{1}{l}{ Lifetime disorder } & & 12-month medication use \\
& Odds ratio (CI) & SE & Odds ratio (CI) \\
\hline $\begin{array}{l}\text { Setting } \\
\text { Community }\end{array}$ & 0.00 & & \\
Care home & $0.212(0.05,0.90) \dagger$ & 0.156 & $8.87(4.99,15.77) \dagger$ \\
Sex & & & \\
Male & 0.00 & & \\
Female & $2.24(1.54,3.26) \dagger$ & 0.430 & $2.18(1.49,3.20) \dagger$ \\
Age & $0.91(0.88,0.94) \dagger$ & 0.016 & $1.01(0.98,1.03)$
\end{tabular}

$\dagger$ Indicates significant differences between community and care home dwellers at the 5\% level 
Table 4: Comparison of health-related quality of life among community and care home dwellers

\begin{tabular}{lll}
\hline & NISHS community sample mean (SD) & Care home sample mean (SD) \\
\hline PCS & $43.8(12.33)$ & $35.1(10.47)$ \\
MCS & $54.02(8.62)$ & $61.6(6.91)$ \\
Physical Functioning $\dagger$ & $1.58(0.82)$ & $2.49(0.78)$ \\
Role Limitations $\dagger$ & $1.64(0.95)$ & $1.84(0.79)$ \\
Social Functioning $\dagger$ & $1.73(1.13)$ & $1.28(0.61)$ \\
Pain $\dagger$ & $2.18(1.24)$ & $1.73(1.10)$ \\
Mental Health $\dagger$ & $1.84(0.99)$ & $1.51(0.81)$ \\
Vitality & $2.74(1.05)$ & $2.84(0.86)$ \\
\hline
\end{tabular}

$\dagger$ Indicates significant differences between community and care home dwellers at the 5\% level

Physical Component Summary (PCS)

Mental Component Summary (MCS)

Table 5: Comparison of SF-6D health related quality of life scores

\begin{tabular}{lll}
\hline & & SF6D Total \\
\hline Community & Mean & 0.78 \\
$\mathrm{n}=852$ & Std. Deviation & 0.16 \\
Care Home & Mean & 0.75 \\
$\mathrm{n}=75$ & Std. Deviation & 0.11 \\
$\mathrm{~T}$ otal & Mean & 0.78 \\
$\mathrm{n}=927$ & Std. Deviation & 0.15 \\
Significant level & & \\
Statistical Test & Community Care Home & 0.556 \\
Kaiser-Meyer-Olkin & 0.853 & \\
measure of & & $76.63 / 15^{*}$ \\
sampling adequacy & $1633 / 15^{*}$ & $39.6 \%$ \\
Bartlett's test of Sphericity & $45 \%$ & 0.63 \\
Variance & 0.82 & $0.75(0.11) *$ \\
Cronbach's alpha & $0.78(0.16)$ & \\
Mean (Std Dev) & & \\
\hline
\end{tabular}

*There was a statistically significant difference between SF6D scores across settings $(t(925) 2.235, \mathrm{P}>0.05)$

Comparison of SF-6D Health Related Quality of Life Scores

The SF-6D score is a preference-based index of overall health-related quality of life ranging from zero to one, derived by applying population-based preference weights to response levels across six dimensions. A higher overall SF6D score indicates better health related quality of life. Examination of the SF6D psychometric properties with factor analysis using maximum likelihood extraction with Promax rotation of the SF6D, indicates that a single factor structure was present. Kaiser-Meyer-Olkin Measure of sampling adequacy was 0.839; Bartlett's test for sphericity $(1617,(\mathrm{df}=15) \mathrm{p}=0.00$ confirm the acceptability of the items for factor analysis. The single factor structure accounted for $52 \%$ of the variance. Cronbach's alpha scores confirm the unidimensionality of the construct (Alpha $=0.81$ ). Therefore, it was appropriate to summate to total level.

A comparison of the average quality of life scores reveals significant differences between the care home sample and NISHS sample (Table 5). Further examination of the association of setting, sex and age with overall health-related quality of life (SF6D score) was conducted using linear regression. Results from linear regression analysis show that females had significantly poorer health-related quality of life than males $(p=0.000)$, controlling for setting and age. There were no significant differences in quality of life scores with respect to setting and age. Therefore, gender was a strong predictor of quality of life scores irrespective of age or setting.

\section{Discussion}

This study examined mental health, quality of life and psychotropic medication use among 75 care home residents and 852 of community dwelling older people. Consistent with other studies (Kessler et al., 2010; O'Connor and Parslow, 2010, McEvoy et al., 2011, Bunting et al., 2012), results from both samples showed a low prevalence of mental disorders among older adults, in comparison to middle-aged and young adults. While there is evidence to suggest that mental disorders are prevalent among nursing home residents (Fullerton et al. (2009); Grabowski et al., 2010), this was not supported in this study. While $18.0 \%$ of individuals over 65 years living in the community met the criteria for any lifetime mood or anxiety disorder, 
just $3.0 \%$ of those in care homes had a lifetime disorder. This is discussed within limitations.

In the context of the age and gender difference between both cohorts, it was found that females were more likely than males to have a mental disorder and older individuals were less likely to have a lifetime mental disorder. This is in keeping with worldwide studies suggesting that while mental health disorders are less prevalent among older age groups, there is a higher prevalence amongst women. An American National Comorbidity Survey undertaken by Byers et al. (2010) found that the likelihood of having a mood, anxiety, or combined mood-anxiety disorder generally showed a pattern of decline with age $(\mathrm{P}<0.05)$. Some evidence from European studies report that for men, depressive mood relates to chronic ill health and for women, the differences are more closely associated with social factors, such as isolation, contact with family and belonging to faith or other community groups (Grundy et al., 2013). Evidence from the United Kingdom points to increasing incidence of depression for men older than 75 years and for women older than 65 years (McCrone et al., 2008). In a Korean study, Kim and Park (2012) found a significant gender difference in the proportion of older men and women with depression. Consistent with the findings of the present study Carayanni et al. (2012) in an urban Greek population found that women had a higher prevalence of depression than men did.

Mental health and quality of life are inextricably linked and this is supported by the results of the present study. Even though health-related quality of life was poorer among the sample of individuals living in care homes and they had significantly poorer physical functioning, participants reported better social functioning, less pain and better mental health than their community dwelling counterparts. These findings are supported by a cross-sectional survey undertaken by Xiao et al. (2016) who explored the relationships between Health-Related Quality of Life (HRQoL), depression and living arrangements among older adults living in both the community $(\mathrm{n}=220)$ and nursing homes $(n=231)$. Results indicated that the older adults' living arrangement did not directly influence their HRQoL but had indirect effects through activities of daily living (ADLs) and depression. This suggests that living in nursing homes does not directly cause older adults' poor HRQoL, but poor ADLs and more severe depression among nursing home residents is likely to lead to poor HRQoL.

While individuals living in care homes were significantly less likely to meet the criteria for a lifetime mental health disorder, they were significantly more likely to have taken mental health medication in the previous 12 months. Females were more likely than males to have taken a mental health medication; however, there was no association between age and mental health medication. Despite the low prevalence of mental disorders reported in the present study, it is significant that over a third $(37.3 \%)(\mathrm{n}=28)$ of the care home residents were being treated for depression. Although this figure is consistent with that of Harris (2012) who found that 37.5 per cent of nursing home residents were prescribed antidepressants, it nonetheless suggests a serious disconnect between diagnosis and prescribed treatment.

It is widely acknowledged that diagnosing depression can be difficult as many of the symptoms (e.g., fatigue, insomnia and reduced appetite) may also be related to other physical health problems (Nicol, 2011). There is also evidence to suggest that depression is commonly inadequately diagnosed and treated in nursing homes (Barca et al., 2009). While this could partially explain the disparity, it could also be argued that the antidepressants were working and therefore participants did not meet the diagnostic criteria for depression. However, our study was based on assessment of mental health disorders at any point in an individual's lifetime and therefore the use of current anti-depressant medication should not have a substantial impact on lifetime prevalence figures. Another possible explanation, reported elsewhere in the literature, is that mental health medication is being employed as a form of chemical restraint (Feng et al., 2009; Stafford, 2010). A recent UK study found that approximately $21 \%$ of care home residents were receiving antipsychotic medications, $80 \%$ of which were prescribed without a diagnosis of mental disorder (Shah et al., 2011). This figure is over twice that reported in the present study $(12 \%)$. Moreover, a recent national data linkage study undertaken within the UK by Maguire et al. (2013) exploring the use of psychotropic medications and the transition into care homes found a higher use of psychotropic drug use in care homes $(20.3 \%)$ compared to the community $(1.1 \%)$. However, regardless of the variation in statistics, it remains the case that some of the care home residents in the present study were on antipsychotic medication without a CIDI (DSM IV) generated diagnosis of a lifetime mood or anxiety disorder.

The use of psychotropic drugs in European nursing homes is reported to be quite frequent (Petek Šter and Cedilnik Gorup, 2011; Mann et al., 2013). Moreover, anxiety and the use of anxiolytics are frequently reported among nursing home residents (Seitz et al., 2010). Results from the present study showed that almost twice as many care home residents $(14.6 \%)(\mathrm{n}=11)$ were taking anxiolytic medication compared to community dwelling older adults (6.5\%). Galik and Resnick (2013) who found that $12 \%$ of nursing residents were taking anxiolytic medication support this finding. The high 
prevalence of psychotropic drug use may indicate that the treatment is not in line with current treatment recommendation (Helvik et al., 2017) suggesting the need for care home staff to monitor the effects and side effects of psychotropic drugs among their residents. The main conclusions of the experimental work should be presented. The contribution of the work to the scientific community and its economic implications should be emphasized.

\section{Limitations}

This study has several limitations for readers to consider. Firstly, the data from both cohorts was not collected concurrently as the NISHS was completed before data were collected from the care home sample. Additionally, it is recognised that the smaller care home sample $(n=75)$ limited a more in-depth analysis of the prevalence of specific mood and anxiety disorders and the association of a wider range of chracteristics with mental health disorders, medication use and QoL in comparison to the larger community dwelling cohort $(n=852)$. However, this provides a rationale for a larger future study examining these key issues in care homes and, in particular, to explore the paradox of low prevalence of mental health disorders but high prescription rates of mental health medication.

Secondly, studies of mood and anxiety disorders based on structured and standardised assessment instruments such as the Composite International Diagnostic Interview (CIDI) are extremely limited (Volkert et al., 2013). Epidemiological studies using the CIDI interview schedule have consistently reported lower prevalence of mental disorders among older adults and this is confirmed in the present study. O'Connor and Paslow (2010) suggested that older adults might respond differently than younger adults to cognitively complex items on the CIDI. However, this could be interpreted as ageist and was later refuted by research, which concluded that significantly lower endorsement of CIDI screening items by older adults was not due to agerelated bias in the items (Buchan et al., 2013).

Thirdly, access to the care home residents was gained through the home managers and they may have put forward their 'best' residents for random selection to portray quality of life in nursing homes in a positive light. Finally, increasing age is associated with increasing frailty and dependence leading to admission to care home. Functional impairment is one of the most important determinants of dependence in older people. It is possible that the care home cohort viewed their new life as one that offered security and protection in a way, they may not have experienced while living at home. The possibility that this may have influenced the findings is acknowledged.

\section{Conclusion}

The population of older adults in long-term care is predicted to increase significantly in the near future. Current government policy across Europe and beyond has seen a shift in emphasis from institutional to community support for older people and given the choice, most older people prefer to remain at home and age in place. This study provides tentative findings to suggest that the mental health and quality of life of socially isolated community dwelling older people may be improved after the move to a care home and this requires consideration by health and social care providers. Conversely, admission to residential care may result in polypharmacy and the administration of psychotropic medication without a DSM IV diagnosis. Further research is required to explore mental health and quality of life among care home residents, with a focus on assessment methods and prescription patterns.

\section{Acknowledgement}

We would like to thank all the study participants and care home managers who assisted with recruitment.

\section{Funding Information}

This study was part of a research fellowship awarded by the Institute of Nursing and Health Research, Ulster University.

\section{Author's Contributions}

Assumpta Ryan and Marie O'Neill: Were responsible for study design, ethical application, drafting and completion of the manuscript. Marie O'Neill undertook data collection.

Paul Slater, Finola Ferry and Brendan Bunting: Were responsible for the data analysis. All authors contributed to the final drafting of the paper.

\section{Ethics}

Ethical approval to conduct the study was granted by the authors' university ethics committee and by the national research ethics committee. The main ethical issues related to the protection of vulnerable adults, participant information, consent, autonomy and confidentiality. The inclusion of home managers as a link between the research team and potential participants afforded additional protection to care home residents. The CIDI interview schedule can take up to $1.5 \mathrm{~h}$ to complete and the research team acknowledged that this may have been very demanding for some participants. Therefore, to minimise participant burden, some interviews were conducted over two visits. 


\section{References}

APA, 1994. Diagnostic and Statistical Manual of Mental Disorders. 4th Edn., American Psychiatric Association, Washington, ISBN-10: 0880486740, pp: 576.

Azermai, M., M. Elseviers, M. Petrovic, L. Van Bortel and R. Stichele, 2011. Assessment of antipsychotic prescribing in Belgian nursing homes. Int. Psychogeriatrics, 23: 1240-1248.

DOI: $10.1017 / \mathrm{S} 104161021100024 \mathrm{X}$

Barcelos-Ferreira, R., E.Y. Nakano, D.C. Steffens and C.M.C. Bottino, 2013. Quality of life and physical activity associated to lower prevalence of depression in community-dwelling elderly subjects from Sao Paulo. J. Affect. Disord. 150: 616-622.

DOI: 10.1016/j.jad.2013.02.024

Beaumont, G. and P. Kenealy, 2004. Quality of life perceptions and social comparisons in healthy old age. Age. Soc., 24: 755-769.

DOI: $10.1017 / \mathrm{s} 0144686 x 04002399$

Barca, M.L., G. Selbæk, J. Laks and K. Engedal, 2009. Factors associated with depression in Norwegian nursing homes. Int. J. Geriatr. Psychiatry, 24: 417-425. DOI: 10.1002/gps.2139

Boorsma, M., K. Joling, M. Dussel, M. Ribbe and D. Frijters et al., 2012. The incidence of depression and its risk factors in Dutch nursing homes and residential care homes. Am. J. Geriatr. Psychiatry, 20: 932-942. DOI: 10.1097/JGP.0b013e31825d08ac

Bowers, H., A. Clark, G. Crosby, L. Easterbrook and A. Macadam et al., 2009. Older people's vision for long-term care. Joseph Rowntree Foundation, New York.

Bowling, A., M. Hankins, G. Windle, C. Bilotta and R. Grant, 2013. A short measure of quality of life in older age: The performance of the brief Older Person's Quality of Life Questionnaire (OPQOLbrief). Archives Gerontol. Geriatr., 56: 181-187. DOI: 10.1016/j.archger.2012.08.012

Bradshaw, S.A., E.D. Playford and A. Riazi, 2012. Living well in care homes: A systematic review of qualitative studies. Age Age., 41: 429-440.

DOI: 10.1093/ageing/afs069

Brazier, J.E. and J.R. Roberts, 2004. The estimation of a preference-based index from the SF-12. Med. Care, 42: 851-859.

Brugha, T., R. Jenkins, N. Taub, H. Meltzer and P. Bebbington, 2001. A general population comparison of the Composite International Diagnostic Interview (CIDI) and the Schedules for Clinical Assessment in Neuropsychiatry ( $S$ CAN). Psychol. Med., 31: 1001-1013. DOI: $10.1017 / \mathrm{S} 0033291701004184$
Buchan, H., M. Sunderland, N. Carragher, P.J. Batterham and T. Slade, 2013. Investigating agerelated differences in responses to screening items for internalising disorders in three national surveys. J. Affect. Disord., 152-154: 229-236.

DOI: 10.1016/j.jad.2013.09.015

Bunting, B.P., S.D. Murphy, S.M. O'Neill and F.R. Ferry, 2012. Lifetime prevalence of mental health disorders and delay in treatment following initial onset: Evidence from the Northern Ireland Study of Health and Stress. Psychol. Med., 42: 1727-1739. DOI: $10.1017 / \mathrm{S} 0033291711002510$

Byers, A.L., K.E. Covinsky, M.B. Friedman and M.L. Bruce, 2010. High occurrence of mood and anxiety disorders among older adults: The national comorbidity survey replication. Arch. Gen. Psychiat., 67: 489-96.

DOI: 10.1001/archgenpsychiatry.2010.35

Carayanni, V., C. Stylianopoulou, G. Koulierakis, F. Babatsikou and C. Koutis, 2012. Sex differences in depression among older adults: Are older women more vulnerable than men in social risk factors? The case of open care centers for older people in Greece. Eur. J. Age., 9: 177-186.

DOI: $10.1007 / \mathrm{s} 10433-012-0216-\mathrm{X}$

Covinsky, K.E., K. Yaffe, K. Lindquist, E. Cherkasova and E. Yelin et al., 2010. Depressive symptoms in middle age and the development of later-life functional limitations: The long-term effect of depressive symptoms. J. Am. Geriatr. Soc., 58: 551-556.

DOI: 10.1111/j.1532-5415.2010.02723.x

Dichter, M.N., M. Halek, O. Dortmann, G. Meyer and S. Bartholomeyczik, 2013. Measuring the quality of life of people with dementia in nursing homes in Germany-the study protocol for the Qol-Dem Project. GMS Psycho-Social-Med., 10: Doc06-Doc06. DOI: $10.3205 / \mathrm{psm} 000096$

Evans, S., C. Gately, P. Huxley, A. Smith and S. Banerjee, 2005. Assessment of quality of life in later life: Development and validation of the QuiLL. Qual. Life Res., 14: 1291-300.

DOI: $10.1007 / \mathrm{s} 11136-004-5532-y$

Feng, Z., J.P. Hirdes, T.F. Smith, H.F. Soveri and I. Chi et al., 2009. Use of physical restraints and antipsychotic medications in nursing homes: A cross-national study. Int. J. Geriatr. Psychiatry, 24: 1110-1118. DOI: $10.1002 / g p s .2232$

Folstein, M.F., S.E. Folstein and P.R. McHugh, 1975. Mini-mental state. A practical method for grading cognitive state of patients for the clinician. J. Psychiatry Res., 12: 189-198.

DOI: $10.1016 / 0022-3956(75) 90026-6$ 
Fullerton, C.A., T.G. McGuire, Z. Feng, V. Mor and D.C. Grabowski, 2009. Trends in mental health admission to nursing homes: 1999-2005. Psychiatr. Serv., 60: 965-971.

DOI: 10.1176/appi.ps.60.7.965

Galik, E. and B. Resnick, 2013. Psychotropic medication use and association with physical and psychosocial outcomes in nursing home residents. J. Psychiatr. Ment. Health Nurs., 20: 244-252.

DOI: $10.1111 / \mathrm{j} .1365-2850.2012 .01911 . x$

Grabowski, D.C., K.A. Aschbrenner, V.F. Rome and S.J. Bartels, 2010. Quality of mental health care for nursing Home residents: A literature review. Med. Care Res. Rev., 67: 627-656.

DOI: $10.1177 / 1077558710362538$

Grundy, E., C. van Campen, D. Deeg, P. Dourgnon and M. Huisman et al., 2013. Health inequalities and the health divide among older people in the WHO European Region: The European review on the social determinants of health and the health divide (Report of the Task Group on Older People). World Health Organization Regional Office for Europe, Copenhagen, Denmark

Hanlon, J.T., X. Wang, N.G. Castle, R.A. Stone and S.M. Handler et al., 2011. Potential underuse, overuse and inappropriate use of antidepressants in older veteran nursing home residents. J. Am. Geriatr. Soc., 59: 1412-1420.

DOI: $10.1111 / \mathrm{j} .1532-5415.2011 .03522 . \mathrm{x}$

Harris, T., I.M. Carey and D. Cook, 2012. Antidepressant prescribing in older primary care patients in the community and care home settings in England and Wales. J. Am. Med. Dir. Assoc., 13: 41-7. DOI: 10.1016/j.jamda.2010.09.005

Haasum, Y., J. Fastbom and K. Johnell, 2012. Institutionalization as a risk factor for inappropriate drug use in the elderly: A Swedish nationwide register-based study. Ann. Pharmacother., 46: 339-346. DOI: 10.1345/aph.1Q597

Harrison, S.L., C. Bradley, R. Milte, E. Liu and L. Kouladjian O’Donnell et al., 2018. Psychotropic medications in older people in residential care facilities and associations with quality of life: A cross-sectional study. BMC Geriatr., 18: 60-60. DOI: $10.1186 / \mathrm{s} 12877-018-0752-0$

Helvik, A.S., J.Š. Benth, B. Wu, K. Engedal and G. Selbæk, 2017. Persistent use of psychotropic drugs in Nursing home residents in Norway. BMC Geriatr., 17: 52-52.

DOI: $10.1186 / \mathrm{s} 12877-017-0440-5$

Hosmer, D.W. and S. Lemeshow, 1989. Applied Logistic Regression. 1st Edn., Wiley, New York, ISBN-10: 0471615536, pp: 307.
James, I., K. Blomberg and A. Kihlgren, 2014. A meaningful daily life in nursing homes-a place of shelter and a space of freedom: A participatory appreciative action reflection study. BMC Nurs., 13: 19-19. DOI: 10.1186/1472-6955-13-19

Kalfoss, M. and L. Halvorsrud, 2009. Important issues to quality of life among Norwegian older adults: An exploratory study. Open Nurs. J., 3: 45-55. DOI: $10.2174 / 1874434600903010045$

Kamble, P., J. Sherer, H. Chen and R. Aparasu, 2010. Off-label use of second-generation antipsychotic agents among elderly nursing home residents. Psychiatr. Serv., 61: 130-136.

DOI: $10.1176 /$ ps.2010.61.2.130

Kessler, R.C. and T.B. Üstun, 2004. The World Mental Health (WMH) survey initiative version of the World Health Organization (WHO) Composite International Diagnostic Interview (CIDI). Int. J. Meth. Psychiatr. Res., 13: 93-121.

DOI: $10.1002 / \mathrm{mpr} .168$

Kessler, R.C. and T.B. Üstun, 2008. The World Health Organization Composite International Diagnostic Interview. In: The WHO World Mental Health Surveys: Global Perspectives on the Epidemiology of Mental Disorders, Kessler, R.C. and T.B. Üstun (Eds.), Cambridge University Press, New York, pp: 58-90.

Kessler, R.C., H.G. Birnbaum, V. Shahly, E. Bromet and I. Hwang et al., 2010. Age differences in the prevalence and co-morbidity of DSM-IV major depressive disorders: Results from the WHO World Mental Health Survey Initiative. Depress. Anxiety, 27: 351-364. DOI: 10.1002/da.20634

Kim, C.G. and S. Park, 2012. Gender difference in risk factors for depression in community-dwelling elders. J. Korean Acad. Nurs., 42: 136-147.

DOI: $10.4040 /$ jkan.2012.42.1.136

Kish, L., 1965. Survey Sampling. 1st Edn., John Wiley, New York, pp: 643.

Luppa, M., C. Sikrski, T. Luck, L. Ehrekem and A. Konnopka et al., 2012. Age-and gender specific prevalence of depression in latest-life-systematic review and meta-analysis. J. Affect. Disord., 136: 212-221. DOI: 10.1016/j.jad.2010.11.033

Maguire, A., C. Hughes, C. Cardwell and D. O'Reilly, 2013. Psychotropic medications and the transition into care: A national data linkage study. J. Am. Geriatr. Soc., 61: 215-221.

DOI: $10.1111 /$ jgs. 12101

Mann, E., B. Haastert, B. Bohmdorfer, T. Fruhwald and B. Iglseder et al., 2013. Prevalence and associations of potentially inappropriate prescriptions in Austrian nursing home residents: Secondary analysis of a cross-sectional study. Wien. Klin. Wochenschr., 125: 180-8. DOI: $10.1007 / \mathrm{s} 00508-013-0342-2$ 
Molzahn, A.E., M. Kalfoss, K.S. Makaroff and S.M. Skevington, 2011. Comparing the importance of different aspects of quality of life to older adults across diverse cultures. Age Age., 40: 192-9.

DOI: $10.1093 /$ ageing/afq156

McCrone, P., S. Dhanasiri, A. Patel, M. Knapp and S. Lawton-Smith, 2008. Paying the Price. The cost of mental health care in England to 2026. The King's Fund, London.

McEvoy, P.M., R. Grove and T. Slade, 2011. Epidemiology of anxiety disorders in the Australian general population: Findings of the 2007 Australian National Survey of Mental Health and Wellbeing. Aust. New Zealand J. Psychiatry, 45: 957-967.

DOI: $10.3109 / 00048674.2011 .624083$

McLafferty, M., S. O’Neill, S. Murphy, C. Armour and F. Ferry et al., 2018. The moderating impact of childhood adversity profiles and conflict on psychological health and suicidal behaviour in the Northern Ireland population. Psychiatry Res., 262: 213-220. DOI: 10.1016/j.psychres.2018.02.024

Naylor, C., M. Parsonage, D. McDaid, M. Knapp and M. Fossey et al., 2012. Long-Term Conditions and Mental Health. The cost of comorbidities. King's Fund and Centre for Mental Health, London, ISBN-10: 9781857176339

Nicol, J., 2011. Nursing Adults with Long-term Conditions (Transforming Nursing Practice Series). 1st Edn., Learning Matters, Exeter, ISBN-10: 0857254421.

O'Connor, D.W. and R.A. Parslow, 2010. Differences in older people's responses to CIDI's depression screening and diagnostic questions may point to age related bias suggest that complex structured psychiatric interviews underestimate rates of mental disorder in old people. J. Affect. Disord., 125: 361-364. DOI: 10.1016/j.jad.2010.01.008

Petek Šter, M. and E. Cedilnik Gorup, 2011. Psychotropic medication use among elderly nursing home residents in Slovenia: Cross-sectional study. Croat. Med. J., 52: 16-24. DOI: $10.3325 / \mathrm{cmj} .2011 .52 .16$

Pitkala, K., A.L. Juola, H. Hosia, M.T. Gronblad and H. Soini et al., 2015. Eight-year trends in the use of opioids, other analgesics and psychotropic medications among institutionalised older people in Finland. J. Am. Med. Dir. Assoc., 16: 973-978. DOI: $10.1016 /$ j.jamda.2015.06.009

RCP, 2014. Depression in older adults. RCP.

Reynolds, K., R.H. Pietrzak, R. El-Gabalawy, C.S. Mackenzie and J. Sareen, 2015. Prevalence of psychiatric disorders in U.S. older adults: Findings from a nationally representative survey. World Psychiatry, 14: 74-81.

DOI: $10.1002 /$ wps.20193
Ruths, S., P.H. Sørensen, O. Kirkevold, B.S. Husebø and K. Krüger et al., 2013. Trends in psychotropic drug prescribing in Norwegian nursing homes from 1997 to 2009: A comparison of six cohorts. Int. J. Geriatr. Psychiatry, 28: 868-876. DOI: $10.1002 / g p s .3902$

Scott, K., M. Von Korff, J. Alonso, M. Angermeyer and E. Bromet et al., 2008. Age patterns in the prevalence of DSM-IV depressive/anxiety disorders with and without physical co-morbidity. Psychol. Med., 38: 1659-1669.

DOI: $10.1017 / \mathrm{S} 0033291708003413$

Seitz, D., N. Purandare and D. Conn, 2010. Prevalence of psychiatric disorders among older adults in longterm care homes: A systematic review. Int. Psychogeriatr., 22: 1025-1039.

DOI: $10.1017 / \mathrm{S} 1041610210000608$

Shah, S., I. Carey, T. Harris, S. Dewilde and D.G. Cook, 2011. Antipsychotic prescribing to older people living in care homes and the community in England and Wales. Int. J. Geriatr. Psychiatry, 26: 423-423. DOI: $10.1002 /$ gps.2557

Shah, S., B. Schoenbachler, J. Streim and S. Meeks, 2012. Antidepressant prescribing patterns in the nursing home: Second-generation issues revisited. J. Am. Med. Dir. Assoc., 13: 406.e13-406.e18.

DOI: 10.1016/j.jamda.2011.09.004

Shruthi, R., R. Jyothi, H.P. Pundarikaksha, G.N. Nagesh and T.J. Tushar, 2016. A Study of medication compliance in geriatric patients with chronic illnesses at a tertiary care hospital. J. Clin. Diagnostic Res., 10: FC40-FC43.

DOI: $10.7860 / J C D R / 2016 / 21908.9088$

Simmons, S.F., K.R. Bonnett, E. Hollingsworth, J. Kim and J. Powers et al., 2017. Reducing antipsychotic medication use in nursing homes: A qualitative study of nursing staff perceptions. The Gerontol., 65: 747-753. DOI: $10.1093 /$ geront/gnx083

Stafford, N., 2010. At least $25 \%$ of elderly residents of German nursing homes are addicted to psychotropic drugs, report claims. BMJ, 340: c2029-c2029.

DOI: 10.1136/bmj.c2029

Tsai, K., J. Chen and C. Wen, 2012. Medication adherence among geriatric outpatients prescribed multiple medications. Am. J. Geriatric Pharmacotherapy, 10: 61-68.

Volkert, J., H. Schulz, M. Harter, O. Wlodarczyk and S. Andreas, 2013. The prevalence of mental disorders in older people in Western countries: A metaanalysis. Age. Res. Rev., 12: 339-353.

DOI: $10.1016 /$ j.arr.2012.09.004 
WHO, 1992. International Classification of Diseases and Related Health Problems. 10th Edn., Switzerland, Geneva.

WHO, 1997. WHOQOL: Measuring quality-of-life. Geneva.

WHO, 2015. World report on ageing and health. WHO. Geneva.

Xiao, H., J.Y. Yoon and B. Bowers, 2016. Living arrangements and quality of life: Mediation by physical function and depression. West J. Nurs. Res., 38: 738-752.

DOI: $10.1177 / 0193945915621260$
Zuidema, S., A. Johansson, G. Selbaek, M. Murray and A. Burns et al., 2015. A consensus guideline for antipsychotic drug use for dementia in care homes. Bridging the gap between scientific evidence and clinical practice. Int. Psychogeriatr., 27: 1849-1859. DOI: $10.1017 / \mathrm{S} 1041610215000745$ 